\title{
Asociación entre actividad física ocupacional y síndrome metabólico: Un estudio poblacional en Perú
}

\section{Association between work-related physical activity and metabolic syndrome: A population-based study in Peru}

\section{RESUMEN}

Existe limitada evidencia de la asociación entre actividad física ocupacional y síndrome metabólico. Esto es importante ya que una gran parte del día cotidiano se gasta en el trabajo, el cual es mayormente sedentario. El objetivo del presente estudio fue evaluar la asociación entre niveles de actividad física ocupacional y la presencia de síndrome metabólico. Método: Análisis de datos secundarios usando información de un estudio poblacional en cinco estratos geográficos del Perú. La variable dependiente fue síndrome metabólico definido según consenso internacional; la exposición fue el auto-reporte de actividad física ocupacional (alto, moderado, bajo). Se usaron modelos de regresión de Poisson reportándose razones de prevalencia $(R P)$ e intervalos de confianza al 95\% (IC95\%). Resultados principales: Datos de 4029 individuos fueron analizados, edad promedio 42,1 años (DE: 15,3) y 2013 (50,0\%) fueron mujeres. Un total de 1011 (25,1\%; IC95\%: 23,8\%-26,5\%) sujetos presentaban síndrome metabólico. El modelo multivariable mostró asociación entre actividad física ocupacional y síndrome metabólico: comparado con los que reportaron altos niveles de actividad física, aquellos con actividad física moderada y baja tenían $R P=1,51$ (IC95\%: 1,25-1,81) y $R P=1,71$ (IC95\%: 1,42-2,06) veces más prevalencia de síndrome metabólico. La asociación fue más fuerte en los varones que en las mujeres $(p=0,001)$. Conclusiones: Nuestros hallazgos confirman la asociación entre niveles de actividad física ocupacional y la presencia de síndrome metabólico. Además 25\% de la población de estudio cumplió con los criterios diagnósticos de síndrome metabólico.

Palabras clave: Actividad física; Dislipidemia; Hiperglucemia; Presión arterial; Prevalencia; Síndrome metabólico.

\footnotetext{
ABSTRACT

There is limited evidence about the association between work-related physical activity and metabolic syndrome. This is important as a great part of a usual day is related to work, and most of this is sedentary. The aim on this study was to assess the association between work-related physical activity and metabolic syndrome. Methods:
}

Valeria Arsentales-Montalva' ${ }^{1}$, María Tenorio-Guadalupe ${ }^{1}$, Antonio Bernabé-Ortiz ${ }^{1,2 *}$

\begin{abstract}
* Dirigir correspondencia a: Antonio Bernabé-Ortiz. CRONICAS Centro de Excelencia en Enfermedades Crónicas, Universidad Peruana Cayetano Heredia, Lima, Perú. Av. Armendáriz 497, Miraflores, Lima 18, Perú. Teléfono: 511-2416978. Email: Antonio.Bernabe@upch.pe
\end{abstract}

Este trabajo fue recibido el 09 de abril de 2018. Aceptado con modificaciones: 13 de abril de 2018 . Aceptado para ser publicado: 07 de enero de 2019.

A secondary analysis of data from a population-based study in five geographical strata in Peru. The outcome of interest was metabolic syndrome based on international consensus; exposure was the self-report of work-related physical activity (high, moderate, and low). Crude and adjusted Poisson regression models, with robust variance were used, from which prevalence ratios $(P R)$ and $95 \%$ confidence intervals $(95 \% \mathrm{CI})$ were reported. Results: Data from 4029 individuals were analyzed, mean age 42.1 (SD: 15.3) years, and 2013 (50.0\%) were females. A total of 1011 (25.1\%; 95\% Cl: 23.8\%-26.5\%) subjects had metabolic syndrome. The multivariable model evidenced an association between work-related physical activity and metabolic syndrome: compared to those reporting 
high levels of physical activity, those with moderate and low physical activity had 1.51 (95\% Cl: 1.25-1.81) and 1.71 (95\% Cl: 1.42-2.06) greater prevalence of metabolic syndrome. The association was stronger among males than females $(p=0.001)$. Conclusions: Our results confirm the association between work-related physical activity and the presence of metabolic syndrome. In addition, $25 \%$ of the study population had metabolic syndrome.

Key words: Blood pressure; Dyslipidemia; Hyperglycemia; Metabolic syndrome; Physical activity; Prevalence.

\section{INTRODUCCIÓN}

El síndrome metabólico (SM) es un conjunto de criterios clínicos que incluyen obesidad abdominal, dislipidemia y niveles de glucosa y presión arterial alterados, asociados a un mayor riesgo de enfermedad cardiovascular, diabetes mellitus tipo 2 (DM-2) y mortalidad en los siguientes 5 a 10 años $^{1,2}$. Durante los últimos años, una serie de estudios han reportado el incremento a nivel mundial del índice de masa corporal $^{3}$, presión arterial ${ }^{4}$, y DM-2 ${ }^{5}$, todos componentes del síndrome metabólico. De otro lado, una reciente revisión sistemática evaluando la prevalencia de SM en la región Asia-Pacífico, reportó que esta podía variar de $12 \%$ hasta 49\% según el país evaluado ${ }^{6}$; mientras que, en América Latina, la prevalencia general de este síndrome fue de casi $25 \%$, variando de $19 \%$ hasta $43 \%$. En Perú, según un estudio realizado en personas de 35 años a más, $47 \%$ presentaba criterios diagnósticos de $\mathrm{SM}^{8}$.

Los factores de riesgo más importantes para el desarrollo de SM son la inactividad física y los patrones de dieta ${ }^{9}$, especialmente aquellos altos en carbohidratos, ya que contribuyen a la aparición de resistencia a la insulina y de obesidad central ${ }^{10}$. Por ejemplo, una revisión sistemática de estudios longitudinales estableció que la dieta mediterránea (rica en frutas y vegetales) tiene un efecto benéfico sobre la progresión a $\mathrm{SM}^{11}$. De otro lado, la realización de actividad física en ratos de ocio ha sido asociada a una reducción de $8 \%$ en el riesgo de SM por cada 10 equivalentes metabólicoshora (MET-hora) por semana de incremento ${ }^{12}$.

La actividad física considera la acumulación diaria de al menos 150 minutos de actividad aeróbica a la semana (o 75 minutos si es actividad aeróbica vigorosa) ${ }^{13}$, incluyendo aquella realizada en momentos de ocio, ocupacionales, o relacionadas con oficios del hogar. Más aun la cantidad de actividad física parece ser diferente entre $\operatorname{sexos}^{14}$. Aunque existe evidencia de asociación entre niveles de actividad física en momentos de ocio y SM, la evidencia es escasa sobre el efecto de la actividad física ocupacional. Esto es importante ya que una gran proporción del tiempo del día se gasta en el trabajo, el cual es mayoritariamente sedentario ${ }^{15,16}$. Por lo tanto, el presente estudio evaluó la asociación entre los niveles de actividad física ocupacional, aquel realizado como parte del trabajo, y la presencia de síndrome metabólico en población peruana adulta. Adicionalmente, se evaluó si el sexo era modificador de efecto de la asociación de interés.

\section{MATERIALES Y MÉTODOS Tipo de Estudio}

El presente es un análisis de datos secundarios usando la información de un estudio de base poblacional Ilamado "Encuesta Nacional de Indicadores Nutricionales, Bioquímicos, Socioeconómicos y Culturales relacionados con las Enfermedades Crónicas no Transmisibles" (ENINBSC), estudio llevado a cabo entre el 2004-2005 ${ }^{17}$. Esta encuesta fue realizada para conocer la prevalencia de enfermedades crónicas no transmisibles, tales como hipertensión arterial, diabetes mellitus tipo 2, dislipidemias, obesidad, entre otras.

\section{Lugar de Estudio}

El estudio fue de base poblacional y se realizó en el territorio peruano, dividiendo al país en cinco estratos poblacionales, determinados según la metodología usual del Instituto Nacional de Estadística e Informática (INEI) ${ }^{17}$. Estos estratos fueron: Lima Metropolitana, resto de la Costa, Sierra urbana, Sierra rural y Selva.

\section{Población de estudio y muestra}

El estudio original tuvo un diseño multietápico. Dentro de cada estrato se aplicó la metodología de selección estandarizada en cuatro etapas: conglomerados, manzanas, viviendas, y personas residentes de las viviendas. De esta forma, la unidad de muestreo fueron las viviendas de los conglomerados seleccionados de acuerdo al peso de cada región según el censo nacional más actualizado, mientras que la unidad de análisis fueron las personas, varones y mujeres de 20 años a más, residentes en cualquiera de los cinco estratos del territorio nacional definidos.

En este estudio se excluyó a las mujeres gestantes y personas que hubieran alterado su dieta habitual por enfermedad. Además, se excluyó a personas con malformaciones físicas o enfermedades graves, así como a personas que practiquen físico-culturismo o algún deporte calificado.

Para el análisis estadístico se incluyó solo a aquellos que tenían los datos completos de actividad física ocupacional y los marcadores completos de SM.

\section{Tamaño de muestra}

Asumiendo un nivel de confianza del $95 \%$ y un poder del $80 \%$, se requerían un total de 1646 participantes para encontrar una asociación (razón de prevalencia) de 1.5 o mayor, dado que $32.2 \%$ de los participantes estaban expuestos (es decir que hacían baja actividad física) y que $13 \%$ del total de la muestra tenía SM dado que tenían altos niveles de actividad física ocupacional. Esta muestra fue duplicada, asumiendo un efecto de diseño de 2 por lo que se requerían 3.292 participantes. Debido a que el total de la población enrolada en el estudio base (ENINBSC) fue de 4.209, se analizó el total de la muestra.

\section{Definición de variables}

Variable dependiente. Fue la presencia de síndrome 
metabólico definido en base al consenso del 2009¹. Según este consenso, se requieren tres o más de los siguientes cinco criterios: (1) obesidad abdominal $(\geq 90 \mathrm{~cm}$ en hombres o $\geq 80 \mathrm{~cm}$ en mujeres); (2) triglicéridos $\geq 150 \mathrm{mg}$ / $\mathrm{dL}$ o hipertrigliceridemia en tratamiento; (3) colesterol $\mathrm{HDL}<40 \mathrm{mg} / \mathrm{dL}$ en varones o $<50 \mathrm{mg} / \mathrm{dL}$ en mujeres; (4) presión arterial $\geq 130 / 85 \mathrm{~mm}$ Hg o hipertensión arterial en tratamiento; y (5) glucosa plasmática en ayunas $\geq 100 \mathrm{mg}$ / $\mathrm{dL}$ o hiperglucemia en tratamiento.

Variable independiente. Fue la actividad física ocupacional, la cual fue auto-reportada y valorada con una pregunta específica sobre la caracterización de la actividad física diaria durante el trabajo. Esta pregunta se basó en la metodología empleada en la Segunda Encuesta Nacional de Factores de Riesgo de $\mathrm{Cuba}^{18}$, pero las preguntas fueron adaptadas a la realidad peruana ${ }^{17}$. De acuerdo a esta pregunta, el participante debía describir la forma en la que dedicaba mayor tiempo en su trabajo. Las opciones de respuesta fueron: (1) usualmente sentado o de pie durante el día y camina poco, (2) camina muchas veces al día, pero no carga objetos muy frecuentemente, (3) usualmente carga y coloca objetos ligeros o sube escaleras frecuentemente, (4) una parte del día realiza poca actividad física y otra parte trabajos fuertes, y (5) hace trabajos fuertes (p. ej. agricultura o carga objetos muy pesados). Para el análisis, esta variable fue categorizada en tres niveles: bajo nivel de actividad física ocupacional (sedentarismo) a la opción de respuesta 1 , nivel moderado de actividad física ocupacional a las opciones de respuesta 2 y 3, y alto nivel de actividad física ocupacional a las opciones de respuesta 4 y 5 .

Otras variables de interés. Por otro lado, se han considerado otras variables del estudio como potenciales confusores de la asociación de interés. Estas fueron: sexo (varón y mujer), edad ( $<40$ años, entre $40-59$ años, y $\geq 60$ años), nivel educativo ( $<7$ años, $7-11$ años, $y \geq 12$ años), estado socioeconómico, creado en base a las posesiones de los participantes y luego categorizado en bajo, medio o alto, y ámbito geográfico (dividido en los 5 estratos territoriales definidos en el muestreo (Lima Metropolitana, resto de la Costa, Sierra rural, Sierra urbana y Selva). Además, se tomaron en cuenta otras variables que constituyen factores de riesgo cardiometabólico como el tabaquismo, basado en el auto-reporte de fumar en los últimos 30 días (si/no) y el consumo de alcohol, categorizado como nunca, sí, pero no en los últimos 30 días, y sí, en los últimos 30 días.

\section{Procedimientos para la recolección de datos}

En el ENINBSC ${ }^{17}$, los entrevistadores visitaron los hogares, y explicaron a los participantes los objetivos del mismo, para obtener el consentimiento informado. Para cada participante, fueron necesarias dos visitas. En la primera se aplicó el cuestionario, mientras que en la segunda se dieron las tomas de muestras sanguíneas en ayunas y las mediciones antropométricas.

La toma de muestras sanguíneas se efectuó por el personal encargado (bioquímico/enfermera) para obtener los valores de glucosa, colesterol total y colesterol HDL. Se extrajeron las muestras en las primeras horas de la mañana luego de un período de ayunas de 8 a 12 horas. Las muestras de sangre fueron obtenidas mediante sistema al vacío con gel activador de la coagulación. Para la obtención del suero se usaron centrífugas manuales (Handzentrifuge) de 3000 RPM y crioviales que permitieron el traslado seguro y conservación de las muestras. Luego las muestras fueron congeladas y enviadas a Lima para análisis en un laboratorio privado, donde fueron analizadas según técnicas estandarizadas. Las muestras de glucosa fueron tratadas en base al método enzimático Trinder-GOD-PAD (glucosa oxidasa), y las de colesterol total y colesterol HDL mediante el método enzimático Trinder-Colorimétrico. De otro lado, las medidas de presión arterial fueron realizadas usando un tensiómetro Mac-Check-501 en la misma visita matinal, mientras que las mediciones antropométricas (talla, peso y circunferencia abdominal) fueron evaluadas utilizando procedimientos estándar.

\section{Plan de análisis de datos}

Los datos del ENINBSC fueron transferidos al programa STATA v13.0 para Windows (Stata Corp, College Station, TX, US) para el análisis. Inicialmente, se realizó la descripción de la población de estudio de acuerdo al nivel de actividad física ocupacional. De otro lado, se estimó la prevalencia de SM y sus respectivos intervalos de confianza al 95\% (IC95\%), y la descripción de la población de estudio fue realizada de acuerdo a la presencia o no de este síndrome. Todas las comparaciones fueron realizadas usando la prueba de Chi cuadrado.

Para evaluar la asociación de interés se utilizaron modelos de regresión de Poisson, con varianza robusta, reportando razones de prevalencia (RP) e IC $95 \%{ }^{19,20}$. Se crearon modelos crudos y ajustados, incluyendo potenciales confusores de acuerdo a la literatura existente ${ }^{21,22}$.

\section{Aspectos éticos}

El protocolo del presente estudio fue aprobado por el Comité de Ética en Investigación de la Universidad Peruana de Ciencias Aplicadas (UPC). La base de datos del ENINBSC es una base que se puede obtener libremente y que se encuentra de-identificada, garantizando la confidencialidad y anonimato de los participantes.

\section{RESULTADOS \\ Descripción de la población de estudio}

El ENINBSC enroló un total de 4.209 participantes, de los cuales sólo se incluyeron a 4.019 (95,5\%) para el presente análisis por tener datos completos en las variables de interés. La edad promedio de los participantes fue de 42,1 años (DE 15,3), 2008 (50,0\%) fueron mujeres, y 1017 (25.3\%) tenían 12 años o más de educación.

Cuando se describió a la población en base a los niveles de actividad física ocupacional, se encontró menor nivel de actividad física ocupacional en mujeres que en varones 
( $p<0.001)$, en aquellos con edad $\geq 60$ años $(p<0.001)$, en aquellos con mayor nivel educativo $(p<0.001)$, con nivel socioeconómico más alto $(p<0.001)$, en aquellos de zona urbana, incluyendo Lima Metropolitana, resto de la Costa y la Sierra urbana $(p<0.001)$, aquellos que reportaron consumo de tabaco $(p<0.001)$ y consumo de alcohol $(p=$ 0.01). Los detalles se encuentran en la Tabla 1.

\section{Prevalencia de síndrome metabólico y factores asociados}

Del total de participantes, 1010 (25,1\%; IC95\%: 23,8\% - 26,5\%) cumplían con los criterios de SM. La prevalencia de SM fue mayor en mujeres que varones $(p<0,001)$, en los de mayor edad $(p<0,001)$, en aquellos con menor nivel de educación $(p=0,004)$, en aquellos con mayor nivel socioeconómico $(p<0,001)$, y en aquellos viviendo en Lima Metropolitana o en el resto de la Costa $(p<0,001)($ Tabla 2$)$.

\section{Actividad física ocupacional y síndrome metabólico}

A menor nivel de actividad física ocupacional, mayor prevalencia de SM $(p<0.001)$. El modelo multivariable mostró evidencia de asociación entre los niveles de actividad física ocupacional y la presencia de SM. Así, comparado contra aquellos que reportaron niveles de actividad física alta, los que reportaron actividad física moderada tenían RP = 1,51 (IC95\%: 1,25 - 1,81) veces más prevalencia de SM. De la misma forma, aquellos que reportaron bajos niveles de actividad física ocupacional tenían RP= 1,71 (IC95\%: 1,42 - 2,06) mayor prevalencia de SM (Tabla 3).

El sexo fue un modificador de efecto de la asociación de interés $(p=0.001)$. Consecuentemente, nuestros análisis mostraron evidencia de una asociación más fuerte entre niveles de actividad física ocupacional y SM en los varones comparado con las mujeres (Tabla 3 ).

\section{DISCUSIÓN Hallazgos principales}

Nuestros hallazgos evidencian una fuerte asociación entre los niveles de actividad física ocupacional y la presencia de síndrome metabólico: comparados con los que reportaron altos niveles de actividad física ocupacional, aquellos con niveles moderado y bajo tenían una mayor prevalencia de SM, incluso después de ajustar por potenciales confusores. De otro lado, el sexo del participante fue un modificador de efecto de la asociación, y por lo tanto, comparado con las mujeres, los varones presentaron mayor probabilidad de SM a similares niveles de actividad física. Finalmente, se encontró que un cuarto de la población evaluada presentaba síndrome metabólico.

\section{Comparación con otros estudios}

Existen estudios previos que avalan el efecto benéfico de la actividad física sobre la salud cardiovascular. Por ejemplo, una revisión sistemática y meta-análisis de siete ensayos clínicos controlados demostró que el ejercicio de resistencia dinámica redujo la circunferencia abdominal y la presión arterial, y aumentó los niveles de colesterol HDL de pacientes con SM, pero no pudo cambiar los niveles de glucosa ni de triglicéridos ${ }^{23}$. De otro lado, otra revisión sistemática, incluyendo estudios de cohorte prospectivos, reportó que altos y moderados niveles de actividad física en momentos de ocio redujeron el riesgo de síndrome metabólico en $20 \%$ y $5 \%$, respectivamente ${ }^{24}$. Sin embargo, los niveles moderados de actividad física solo fueron significativos en los varones y no en mujeres. Otros estudios, de naturaleza transversal han encontrado similares hallazgos ${ }^{9,21,25}$.

Sin embargo, es poca la evidencia que se encuentra sobre el efecto de la actividad física ocupacional en la probabilidad de presentar síndrome metabólico. Más aun, algunas de ellas presentan resultados contradictorios. Por ejemplo, un estudio transversal de empleados en Suecia ${ }^{25}$, reportó que la actividad física ocupacional vigorosa redujo en 34\% la prevalencia de hipertensión y en $47 \%$ la prevalencia de colesterol HDL bajo, pero no afecto la hipercolesterolemia. De otro lado, otro estudio transversal en varones y mujeres de 60 años a más realizado en la misma Suecia ${ }^{22}$, solo encontró asociación entre actividad física en momentos de ocio, pero no aquella relacionada con el trabajo, y síndrome metabólico. Otro estudio de base poblacional realizado en Francia encontró que la actividad física ocupacional estuvo asociada a SM pero solo en mujeres y no en varones ${ }^{9}$. Finalmente, otro estudio en Perú mostró que aquellos en altos niveles de actividad física en momentos de ocio tenían una reducción del $42 \%$ en el riesgo de síndrome metabólico ${ }^{26}$. Nuestro estudio no tan solo establece la asociación de interés estudiada, sino que además remarca que los varones presentaron mayor probabilidad de SM a similares niveles de actividad física ocupacional que las mujeres. De acuerdo a eso, mientras que bajos niveles de actividad física ocupacional en mujeres incrementa en 35\% la probabilidad de síndrome metabólico, esta probabilidad Ilega hasta $86 \%$ en los varones. Esto probablemente pueda estar asociado al hecho de que los varones realizan un trabajo más sedentario que las mujeres.

Nuestros hallazgos también muestran que aproximadamente uno de cada cuatro habitantes de 20 años a mas presentan síndrome metabólico en Perú. Aunque hay varios estudios previos en la literatura ${ }^{27,28,29,30,31}$, muchos de ellos enrolaron poblaciones específicas o de altura. Más aun, se ha descrito que muchos de ellos fallan en el reporte de la metodología o los resultados ${ }^{32}$, por lo que es necesario estudios que involucren a toda la población peruana.

\section{Relevancia en Salud Pública}

Se debe establecer estrategias de intervención apropiadas orientadas a la prevención del síndrome metabólico como una prioridad en la atención sanitaria y al nivel del lugar de trabajo, centrados sobretodo en la modificación de los estilos de vida, incluyendo una dieta saludable y actividad física.

Existe evidencia de que la actividad física es una herramienta clínica efectiva para la reducción de riesgo 
Tabla 1. Descripción de población estudio según actividad física ocupacional.

\begin{tabular}{|c|c|c|c|c|}
\hline & $\begin{array}{c}\text { Alto }_{N}^{\text {Nive }} \\
N=1107\end{array}$ & $\begin{array}{l}\text { Moderado } \\
N=1626\end{array}$ & 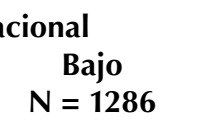 & p-valor \\
\hline \multicolumn{5}{|l|}{ Sexo } \\
\hline Mujer & $285(25,7 \%)$ & $1014(62,4 \%)$ & 709 (55,1\%) & $<0.001$ \\
\hline Varón & $822(74,3 \%)$ & $612(37,6 \%)$ & 577 (44,9\%) & \\
\hline \multicolumn{5}{|l|}{ Edad* } \\
\hline$<40$ años & $528(47,7 \%)$ & $834(51,3 \%)$ & $619(48,1 \%)$ & $<0.001$ \\
\hline 40 - 59 años & $437(39,5 \%)$ & $576(35,4 \%)$ & $412(32,0 \%)$ & \\
\hline$\geq 60$ años & $142(12,8 \%)$ & $216(13,3 \%)$ & $255(19,9 \%)$ & \\
\hline \multicolumn{5}{|l|}{ Nivel educativo* } \\
\hline$<7$ años & $589(53,2 \%)$ & $535(32,9 \%)$ & $437(34,0 \%)$ & $<0.001$ \\
\hline $7-11$ años & $394(35,6 \%)$ & $580(35,6 \%)$ & $466(36,3 \%)$ & \\
\hline$\geq 12$ años & $124(11,2 \%)$ & $511(31,4 \%)$ & $382(29,7 \%)$ & \\
\hline \multicolumn{5}{|l|}{ Nivel socioeconómico } \\
\hline Bajo & $651(58,8 \%)$ & $432(26,6 \%)$ & $308(24,0 \%)$ & $<0.001$ \\
\hline Medio & $329(29,7 \%)$ & $616(37,9 \%)$ & $507(39,4 \%)$ & \\
\hline Alto & $127(11,5 \%)$ & $578(35,6 \%)$ & $471(36,6 \%)$ & \\
\hline \multicolumn{5}{|l|}{ Ámbito geográfico } \\
\hline Lima Metropolitana & $71(6,4 \%)$ & $397(24,4 \%)$ & $343(26,7 \%)$ & $<0.001$ \\
\hline Resto de Costa & $171(15,4 \%)$ & $317(19,5 \%)$ & $272(21,2 \%)$ & \\
\hline Selva & $264(23,9 \%)$ & $294(18,1 \%)$ & $242(18,8 \%)$ & \\
\hline Sierra Rural & $425(38,4 \%)$ & $244(15,0 \%)$ & $140(10,9 \%)$ & \\
\hline Sierra Urbana & $176(15,9 \%)$ & $374(23,0 \%)$ & $289(22,5 \%)$ & \\
\hline \multicolumn{5}{|l|}{ Tabaquismo* } \\
\hline No & $846(76,8 \%)$ & $1371(84,8 \%)$ & $1093(85,1 \%)$ & $<0.001$ \\
\hline $\mathrm{Si}$ & $256(23,2 \%)$ & $246(15,2 \%)$ & $191(14,9 \%)$ & \\
\hline \multicolumn{5}{|l|}{ Consumo de alcohol* } \\
\hline Nunca & $63(5,7 \%)$ & $117(7,2 \%)$ & $82(6,4 \%)$ & 0.01 \\
\hline Sí, no últimos 30 días & $472(42,7 \%)$ & $770(47,4 \%)$ & $620(48,2 \%)$ & \\
\hline Si, en últimos 30 días & $570(51,6 \%)$ & 739 (45,4\%) & $583(45,4 \%)$ & \\
\hline
\end{tabular}

* Los valores pueden no sumar debido a valores faltantes.

cardiovascular, incluido el síndrome metabólico ${ }^{12}$. Se ha demostrado que el entrenamiento físico disminuye el peso corporal y la circunferencia abdominal, mejora la sensibilidad a la insulina, aumenta los niveles circulatorios del colesterol HDL y normaliza la presión arterial, componentes de este síndrome ${ }^{23,24}$.

Dado el efecto benéfico de la actividad física, diversas organizaciones a nivel del mundo han implementado la realización de actividad física dentro de la jornada laboral obteniendo resultados positivos tanto en la salud física de los trabajadores como en la productividad de la empresa. Por ejemplo, una revisión sistemática de intervenciones encontró que las intervenciones en el lugar de trabajo incrementaron los minutos por semana de equivalentes metabólicos con la subsecuente reducción en la masa corporal, el índice de masa corporal, el colesterol LDL y la glucosa en sangre ${ }^{33}$. Sin embargo, un estudio anterior estableció que la implementación de consejería sobre actividad física en el puesto de trabajo no era costo-efectivo en el corto plazo, aunque podría favorecer el gasto de energía ${ }^{34}$. Si las intervenciones deberían ser específicas de sexo/género es algo que aún no se ha establecido. Sin embargo, diversos factores cardiovasculares son más frecuentes en varones (ej. tabaquismo y consumo de alcohol), mientras que 
Tabla 2. Descripción de la población de acuerdo a la presencia de síndrome metabólico.

\begin{tabular}{|c|c|c|c|}
\hline & $\begin{array}{c}\text { Síndrome } M \\
\text { No } \\
\mathrm{N}=3009\end{array}$ & $\stackrel{\substack{\text { ico } \\
N=1010}}{ }$ & p-valor \\
\hline \multicolumn{4}{|l|}{ Sexo } \\
\hline Femenino & $1327(44,1 \%)$ & $681(67,4 \%)$ & \multirow[t]{2}{*}{$<0.001$} \\
\hline Masculino & $1682(55,9 \%)$ & $329(32,6 \%)$ & \\
\hline \multicolumn{4}{|l|}{ Edad* } \\
\hline$<40$ años & 1707 (56,7\%) & $274(27,1 \%)$ & \multirow[t]{3}{*}{$<0.001$} \\
\hline $40-59$ años & $936(31,1 \%)$ & $489(48,4 \%)$ & \\
\hline$>60$ años & $366(12,2 \%)$ & $247(24,5 \%)$ & \\
\hline \multicolumn{4}{|l|}{ Nivel educativo* } \\
\hline$<7$ años & $1126(37,4 \%)$ & $435(43,1 \%)$ & \multirow[t]{3}{*}{0.004} \\
\hline 7 - 11 años & $1092(36,3 \%)$ & $348(34,4 \%)$ & \\
\hline$>12$ años & $790(26,3 \%)$ & $227(22,5 \%)$ & \\
\hline \multicolumn{4}{|l|}{ Nivel socioeconómico } \\
\hline Bajo & $1135(37,7 \%)$ & $256(25,4 \%)$ & \multirow[t]{3}{*}{$<0.001$} \\
\hline Medio & $1088(36,2 \%)$ & $364(36,0 \%)$ & \\
\hline Alto & $786(26,1 \%)$ & $390(38,6 \%)$ & \\
\hline \multicolumn{4}{|l|}{ Ámbito geográfico } \\
\hline Lima Metropolitana & $571(19,0 \%)$ & $240(23,7 \%)$ & \multirow[t]{5}{*}{$<0.001$} \\
\hline Resto de Costa & $527(17,5 \%)$ & $233(23,1 \%)$ & \\
\hline Selva & $603(20,0 \%)$ & 197 (19,5\%) & \\
\hline Sierra rural & $668(22,2 \%)$ & $141(14,0 \%)$ & \\
\hline Sierra urbana & $640(21,2 \%)$ & 199 (19.7\%) & \\
\hline \multicolumn{4}{|l|}{ Tabaquismo* } \\
\hline No & $2436(81,3 \%)$ & $874(86,8 \%)$ & \multirow[t]{2}{*}{$<0.001$} \\
\hline $\mathrm{Si}$ & $560(18,7 \%)$ & $133(13,2 \%)$ & \\
\hline \multicolumn{4}{|l|}{ Consumo de alcohol* } \\
\hline Nunca & $195(6,5 \%)$ & $67(6,6 \%)$ & \multirow[t]{3}{*}{$<0.001$} \\
\hline Sí, no últimos 30 días & $1341(44,6 \%)$ & $521(51,6 \%)$ & \\
\hline Si, en últimos 30 días & $1470(48,9 \%)$ & $422(41,8 \%)$ & \\
\hline
\end{tabular}

* Los valores pueden no sumar debido a valores faltantes.

otros son más frecuentes en mujeres (ej. obesidad, bajo colesterol HDL).

\section{Fortalezas y Limitaciones}

Nuestro estudio cuenta con una muestra representativa, y de diferentes estratos de las diversas regiones del Perú. Asimismo, evalúa al sexo como modificador de efecto de la asociación entre actividad física ocupacional y síndrome metabólico. Sin embargo, este estudio tiene algunas limitaciones. En primer lugar, debido a ser un estudio transversal, solo evalúa asociación, y no causalidad. En segundo lugar, la evaluación de actividad física ocupacional no es la usual o la utilizada en otros estudios como el modulo respectivo del Cuestionario Internacional de Actividad Física (IPAQ). En tercer lugar, cierta información, aquella referida a estilos de vida, podría verse afectada por el sesgo de deseabilidad social, lo que podría subestimar la real prevalencia de factores de riesgo como en el caso de tabaquismo o alcohol. Finalmente, no se incluyó ciertas variables confusoras como por ejemplo patrones de dieta, así como causas secundarias de síndrome metabólico, como enfermedades endocrinas o de base farmacológica como la 
Tabla 3. Asociación entre niveles de actividad física ocupacional y síndrome metabólico: modelos crudos y ajustados.

\begin{tabular}{|c|c|c|c|c|}
\hline $\begin{array}{l}\text { Niveles de actividad física } \\
\text { ocupacional }\end{array}$ & \multicolumn{2}{|c|}{ Síndrome Metabólico } & $\begin{array}{l}\text { Modelo crudo } \\
\text { RP (IC95\%) }\end{array}$ & $\begin{array}{l}\text { Modelo ajustado } \\
\text { RP (IC95\%) }\end{array}$ \\
\hline Para toda la muestra & $(\mathrm{N}=3009)$ & $(\mathrm{N}=1010)$ & & \\
\hline Alto & $963(87,0 \%)$ & $144(13,0 \%)$ & 1 (Referencia) & 1 (Referencia) \\
\hline Medio & $1174(72,2 \%)$ & $452(27,8 \%)$ & $2,14(1,80-2,54)$ & $1,51(1,25-1,81)$ \\
\hline Bajo & $872(67,8 \%)$ & $414(32,2 \%)$ & $2,47(2,08-2,94)$ & $1,71(1,42-2,07)$ \\
\hline En mujeres & $(\mathrm{N}=1327)$ & $(\mathrm{N}=681)$ & & \\
\hline Alto & 213 (74,7\%) & $72(25,3 \%)$ & 1 (Referencia) & 1 (Referencia) \\
\hline Medio & $686(67,6 \%)$ & $328(32,4 \%)$ & $1,28(1,03-1,60)$ & $1,20(0,97-1,49)$ \\
\hline Bajo & $428(60,4 \%)$ & $281(39,6)$ & $1,57(1,26-1,95)$ & $1,35(1,08-1,69)$ \\
\hline En varones & $(\mathrm{N}=1682)$ & $(\mathrm{N}=329)$ & & \\
\hline Alto & 750 (91,2\%) & $72(8.8 \%)$ & 1 (Referencia) & 1 (Referencia) \\
\hline Medio & 488 (79,7\%) & $124(20,3 \%)$ & $2,31(1,76-3,03)$ & $1,62(1,20-2,20)$ \\
\hline Bajo & $444(77,0 \%)$ & $133(23,0 \%)$ & $2,63(2,02-3,43)$ & $1,86(1,37-2,53)$ \\
\hline
\end{tabular}

* Ajustado por sexo, edad, nivel educativo, nivel socioeconómico, ámbito geográfico, tabaquismo y consumo de alcohol.

corticoterapia crónica. Sin embargo, siendo este un estudio poblacional, el potencial efecto de estas enfermedades podría ser casi nulo sobre los resultados mostrados.

\section{CONCLUSIONES}

Nuestros hallazgos confirman la asociación entre niveles de actividad física ocupacional y la presencia de síndrome metabólico. Se observó además que 25\% de la población de estudio cumplió con los criterios diagnósticos de síndrome metabólico. Nuestros hallazgos sugieren que es de importancia crítica abordar los factores de riesgo cardiovasculares como una prioridad de la atención sanitaria, especialmente entre la población que reporta menor nivel de actividad física.

Conflictos de interés: Los autores declaran no tener conflictos de interés.

\section{BIBLIOGRAFÍA}

1. Alberti KG, Eckel RH, Grundy SM, et al. Harmonizing the metabolic syndrome: a joint interim statement of the International Diabetes Federation Task Force on Epidemiology and Prevention; National Heart, Lung, and Blood Institute; American Heart Association; World Heart Federation; International Atherosclerosis Society; and International Association for the Study of Obesity. Circulation 2009; 120: 1640-1645.

2. Kaur J. A comprehensive review on metabolic syndrome. Cardiol Res Pract 2014; 2014: 943162.

3. NCD Risk Factor Collaboration. Worldwide trends in bodymass index, underweight, overweight, and obesity from 1975 to 2016: a pooled analysis of 2416 population-based measurement studies in 128.9 million children, adolescents, and adults. Lancet 2017; 390: 2627-2642.

4. NCD Risk Factor Collaboration. Worldwide trends in blood pressure from 1975 to 2015: a pooled analysis of 1479 population-based measurement studies with 19.1 million participants. Lancet 2017; 389: 37-55.

5. NCD Risk Factor Collaboration. Worldwide trends in diabetes since 1980: a pooled analysis of 751 population-based studies with 4.4 million participants. Lancet 2016; 387: 1513-1530.

6. Ranasinghe $P$, Mathangasinghe $Y$, Jayawardena $R$, Hills $A P$, Misra A. Prevalence and trends of metabolic syndrome among adults in the asia-pacific region: a systematic review. BMC Public Health 2017; 17: 101.

7. Marquez-Sandoval F, Macedo-Ojeda G, Viramontes-Horner $D$, Fernandez Ballart JD, Salas Salvado J, Vizmanos B. The prevalence of metabolic syndrome in Latin America: a systematic review. Public Health Nutr 2011;1 4: 1702-1713.

8. Bernabe-Ortiz A, Carrillo-Larco RM, Gilman RH, Checkley W, Smeeth L, Miranda JJ. Contribution of modifiable risk factors for hypertension and type-2 diabetes in Peruvian resourcelimited settings. I Epidemiol Community Health 2016; 70: 49-55.

9. Wagner A, Dallongeville J, Haas B, et al. Sedentary behaviour, physical activity and dietary patterns are independently associated with the metabolic syndrome. Diabetes Metab 2012; 38: 428-435.

10. Adochio RL, Leitner JW, Gray K, Draznin B, Cornier MA. Early responses of insulin signaling to high-carbohydrate and high-fat overfeeding. Nutr Metab 2009; 6: 37.

11. Esposito K, Kastorini CM, Panagiotakos DB, Giugliano D. Mediterranean diet and metabolic syndrome: an updated systematic review. Rev Endocr Metab Disord 2013; 14: 255263.

12. Zhang D, Liu X, Liu $Y$, et al. Leisure-time physical activity and incident metabolic syndrome: a systematic review and 
dose-response meta-analysis of cohort studies. Metabolism 2017; 75: 36-44.

13. National Health Service. Physical activity guidelines for adults (19-64 years). London, UK: NHS; 2011.

14. O'Neil A, Scovelle AJ, Milner AJ, Kavanagh A. Gender/Sex as a Social Determinant of Cardiovascular Risk. Circulation 2018; 137: 854-864.

15. Popkin BM, Adair LS, Ng SW. Global nutrition transition and the pandemic of obesity in developing countries. Nutr Rev 2012; 70: 3-21.

16. Pulakka A, Stenholm S, Bosma H, et al. Association Between Employment Status and Objectively Measure d Physical Activity and Sedentary Behavior-The Maastricht Study. I Occup Environ Med 2018; 60: 309-315.

17. Instituto Nacional de Salud. Encuesta Nacional de Indicadores Nutricionales, Bioquimicos, Socioeconomicos y Culturales relacionados con las Enfermedades Cronicas Degenerativas. Lima, Peru: Instituto Nacional de Salud: Centro Nacional de Alimentacion y Nutricion; 2006.

18. Cabrera $A$, Jimenez $S$, Hernandez $M$, Quintero $M$, Diaz $M$, Romero M, Ferret A, Wong I, Moreno V. Algunos factores de riesgo a enfermedades cardiovasculares en un grupo de adultos supuestamente sanos. Rev Cubana Aliment Nutr 1997; 11(1): 40-45.

19. Barros AJ, Hirakata VN. Alternatives for logistic regression in cross-sectional studies: an empirical comparison of models that directly estimate the prevalence ratio. BMC Med Res Methodol 2003; 3: 21.

20. Coutinho LM, Scazufca M, Menezes PR. Methods for estimating prevalence ratios in cross-sectional studies. Rev Saude Publica 2008; 42: 992-998.

21. Cho ER, Shin A, Kim J, Jee SH, Sung J. Leisure-time physical activity is associated with a reduced risk for metabolic syndrome. Ann Epidemiol 2009; 19: 784-792.

22. Halldin M, Rosell M, de Faire U, Hellenius ML. The metabolic syndrome: prevalence and association to leisure-time and work-related physical activity in 60-year-old men and women. Nutrition, metabolism, and cardiovascular diseases. Nutr Metab Cardiovasc Dis 2007; 17: 349-357.

23. Pattyn N, Cornelissen VA, Eshghi SR, Vanhees L. The effect of exercise on the cardiovascular risk factors constituting the metabolic syndrome: a meta-analysis of controlled trials. Sports Med 2013; 43: 121-133.

24. He D, Xi B, Xue J, Huai P, Zhang M, Li J. Association between leisure time physical activity and metabolic syndrome: a meta-analysis of prospective cohort studies. Endocrine 2014; 46: 231-240.

25. Fransson El, Alfredsson $L S$, de Faire UH, Knutsson A, Westerholm PJ. Leisure time, occupational and household physical activity, and risk factors for cardiovascular disease in working men and women: the WOLF study. Scand J Public health 2003; 31: 324-333.

26. Gelaye B, Revilla L, Lopez $T$, Sanchez $S$, Williams MA. Prevalence of metabolic syndrome and its relationship with leisure time physical activity among Peruvian adults. Eur I Clin Invest 2009; 39: 891-898.

27. Baracco R, Mohanna S, Seclen S. A comparison of the prevalence of metabolic syndrome and its components in high and low altitude populations in peru. Metab Syndr Relat Disord 2007; 5: 55-62.

28. Bernabe-Ortiz A, Benziger CP, Gilman RH, Smeeth L, Miranda J). Sex differences in risk factors for cardiovascular disease: the PERU MIGRANT study. PLoS One 2012;7: e35127.

29. Medina-Lezama J, Zea-Diaz H, Morey-Vargas OL, et al. Prevalence of the metabolic syndrome in Peruvian Andean hispanics: the PREVENCION study. Diabetes Res Clin Pract 2007; 78: 270-281.

30. Ninatanta-Ortiz JA, Nunez-Zambrano LA, Garcia-Flores SA, Romani FR. [Frequency of metabolic syndrome in residents of an andean region in Peru]. Rev Perú Med Exp Salud Publica 2016; 33: 640-650.

31. Seclen S, Villena A, Larrad MT, et al. Prevalence of the metabolic syndrome in the mestizo population of Peru. Metab Syndr Relat Disord 2006; 4: 1-6.

32. Tapia JC, Ruiz EF, Ponce OJ, Malaga G, Miranda J. Weaknesses in the reporting of cross-sectional studies according to the STROBE statement: the case of metabolic syndrome in adults from Peru. Colomb Med 2015; 46: 168-175.

33. Reed IL, Prince SA, Elliott CG, et al. Impact of Workplace Physical Activity Interventions on Physical Activity and Cardiometabolic Health Among Working-Age Women: A Systematic Review and Meta-Analysis. Circ Cardiov Qual Outcomes 2017; 10, pii: e003516

34. Proper KI, de Bruyne MC, Hildebrandt VH, van der Beek AJ, Meerding WJ, van Mechelen W. Costs, benefits and effectiveness of worksite physical activity counseling from the employer's perspective. Scand J Work Environ Health 2004; 30: 36-46. 